\title{
SOLVABILITY OF THE GENERALIZED POSSIO EQUATION IN 2D SUBSONIC AEROELASTICITY.
}

\author{
PETER L. POLYAKOV
}

\begin{abstract}
We study solvability of the generalized Possio integral equation - a tool in analysis of a boundary value problem in $2 \mathrm{D}$ subsonic aeroelasticity with the Kutta-Joukowski condition "zero pressure discontinuity" - $\psi(x, 0, t)=0$ on the complement of a finite interval in the whole real line $\mathbb{R}$. The corresponding problem with boundary condition on finite intervals adjacent to the "chord" was considered in $[\mathrm{P}]$.
\end{abstract}

\section{InTRODUCTION.}

We consider the linearized subsonic inviscid compressible flow equation in $2 \mathrm{D}$ ([Se], $[\mathrm{BAH}],[\mathrm{Ba} 2])$

$$
a^{2}\left(1-M^{2}\right) \frac{\partial^{2} \phi}{\partial x^{2}}+a^{2} \frac{\partial^{2} \phi}{\partial y^{2}}=\frac{\partial^{2} \phi}{\partial t^{2}}+2 M a \frac{\partial^{2} \phi}{\partial t \partial x}
$$

where $a$ is the speed of sound, $M=\frac{U}{a}<1$ - the Mach number, $U$ - free stream velocity, $\phi(x, y, t)$ - small disturbance velocity potential, considered on

$$
\left\{(x, y, t):(x, y) \in \mathbb{R}^{2} \backslash\{x \in[-1,1], y=0\}, 0 \leq t<\infty\right\},
$$

with boundary conditions:

- flow tangency condition

$$
\frac{\partial \phi}{\partial y}(x, 0, t)=w(x, t),|x|<b
$$

where $b$ is the "half-chord", and $w$ is the given normal velocity of the wing, without loss of generality we will assume in what follows that $b=1$,

- "strong Kutta-Joukowski condition" for the acceleration potential

$$
\begin{aligned}
& \psi(x, y, t):=\frac{\partial \phi}{\partial t}+U \frac{\partial \phi}{\partial x}, \\
& \psi(x, 0, t)=0 \text { for }|x|>1,
\end{aligned}
$$

- far field condition

$$
\phi(x, y, t) \rightarrow 0, \text { as }|x| \rightarrow \infty \text {, or } y \rightarrow \infty .
$$

In $[\mathrm{P}]$ we considered a weaker form of the boundary condition (3), requiring for acceleration potential to be zero not on the whole $\mathbb{R} \backslash[-1,1]$, but only on finite intervals adjacent to the interval $[-1,1]$. In the present paper we address the problem with "auxiliary boundary conditions" from $([\mathrm{BAH}]$, p. 319) on the whole $\mathbb{R} \backslash[-1,1]$, combining technical tools from $[\mathrm{P}]$ with Possio's approach as it is described in the fascinating book $[\mathrm{BAH}]$. We also

Date: August 26, 2018.

1991 Mathematics Subject Classification. 45B05,45E05.

Key words and phrases. Reduced wave equation, Finite Hilbert transform, Fredholm determinant. 
generalize the Possio's scheme of construction of a solution of the boundary value problem (1), (2), (3) by including the case of arbitrary (not only harmonic) dependence of $w(x, t)$ (and consequently of a solution) on time.

Before formulating the main result of the article we introduce a couple of notations. For a function $w(x, t)$ on $[-1,1] \times \mathbb{R}^{+}$we denote by

$$
\widehat{w}(x, s)=\int_{0}^{\infty} e^{-s t} w(x, t) d t,
$$

the Laplace transform of $w(x, t)$, an analytic function of $s=\sigma+i \nu$ in the half-plane $\left\{s: \operatorname{Re} s>\sigma_{0}\right\}$. In section 6 we introduce function $D_{N}(s)$ (formula (33) and Proposition 6.2), analytic in the same half-plane and depending only on function $H_{0}^{(1)}$ - the Hankel function of the first kind of order 0.

The main result of the article is the theorem below.

Theorem 1. Let function $D_{N}(s)$ from formula (33), mentioned above, have no zeros in the strip $\left\{s:\right.$ Res $\left.\in\left[\sigma_{1}, \sigma_{2}\right]\right\}$, where $\sigma_{1}>\sigma_{0}$. Let $w(\cdot, t) \in L^{2}[-1,1]$ be such that for some $\epsilon>0$

$$
\|\widehat{w}(\cdot, \sigma+i \nu)\|_{L^{2}[-1,1]}<\exp \left\{-e^{|\nu|} \cdot(1+|\nu|)^{4+\epsilon}\right\} \text { for } \sigma \in\left[\sigma_{1}, \sigma_{2}\right]
$$

Then equation (1) has a solution of the form

$$
\phi(x, y, t)=-\frac{1}{2 \pi i} \int_{\sigma^{\prime}-i \infty}^{\sigma^{\prime}+i \infty} d s e^{s(t+c x)} \frac{e^{\lambda(s) x}}{U} \int_{-1}^{1} p(\xi, s) d \xi \int_{-\infty}^{x} e^{-\lambda(s) u}\left(\left.\frac{\partial H_{0}^{(1)}}{\partial y}(\zeta)\right|_{\eta=0}\right) d u
$$

where $c=\frac{M}{a\left(1-M^{2}\right)}, \lambda(s)=-\frac{s(1+c U)}{U}, H_{0}^{(1)}$ - the Hankel function of the first kind of order 0 , and

$$
\zeta=\frac{i s}{a \sqrt{1-M^{2}}} \sqrt{\frac{(u-\xi)^{2}}{1-M^{2}}+(y-\eta)^{2}} .
$$

This solution is independent of $\sigma^{\prime} \in\left[\sigma_{1}, \sigma_{2}\right]$, satisfies boundary conditions above, and function $p(\xi, s)$ satisfies the estimate

$$
\int_{-1}^{1}\left|p\left(\xi, \sigma^{\prime}+i \nu\right)\right|^{p} d \xi<C
$$

with $C>0$ independent of $s$ for arbitrary $p<\frac{4}{3}$.

\section{Particular solution of equation (1).}

In this section we follow Possio's idea of constructing a special solution of equation (1) that represents the acceleration potential. It is easy to see that since equation (1) is linear, the acceleration potential, defined in the linear model of subsonic flow by formula in (3) also satisfies equation (1). In order to construct this special solution of differential equation (1) we use two lemmas below.

Lemma 2.1. If function $\Psi(x, y)$ satisfies the reduced wave equation

$$
a^{2}\left(1-M^{2}\right) \frac{\partial^{2} \Psi}{\partial x^{2}}(x, y)+a^{2} \frac{\partial^{2} \Psi}{\partial y^{2}}(x, y)-\frac{s^{2}}{1-M^{2}} \Psi(x, y)=0
$$

then function

$$
\psi(x, y, t)=\Psi(x, y) e^{s(t+c x)}
$$


with

$$
c=\frac{M}{a\left(1-M^{2}\right)}
$$

satisfies equation (1).

Proof. For $\psi$ defined in (8) we have

$$
\begin{gathered}
a^{2}\left(1-M^{2}\right) \frac{\partial^{2} \psi}{\partial x^{2}}+a^{2} \frac{\partial^{2} \psi}{\partial y^{2}}-\frac{\partial^{2} \psi}{\partial t^{2}}-2 M a \frac{\partial^{2} \psi}{\partial t \partial x} \\
=e^{s(t+c x)}\left[a^{2}\left(1-M^{2}\right)\left(\frac{\partial^{2} \Psi}{\partial x^{2}}(x, y)+2 s c \frac{\partial \Psi}{\partial x}(x, y)+s^{2} c^{2} \Psi(x, y)\right)+a^{2} \frac{\partial^{2} \Psi}{\partial y^{2}}(x, y)\right. \\
\left.-s^{2} \Psi(x, y)-2 M a\left(s \frac{\partial \Psi}{\partial x}(x, y)+s^{2} c \Psi(x, y)\right)\right] \\
=e^{s(t+c x)}\left[a^{2}\left(1-M^{2}\right) \frac{\partial^{2} \Psi}{\partial x^{2}}(x, y)+a^{2} \frac{\partial^{2} \Psi}{\partial y^{2}}(x, y)\right. \\
\left.+\left(a^{2}\left(1-M^{2}\right) s^{2} c^{2}-2 M a s^{2} c-s^{2}\right) \Psi(x, y)\right] \\
=e^{s(t+c x)}\left[a^{2}\left(1-M^{2}\right) \frac{\partial^{2} \Psi}{\partial x^{2}}(x, y)+a^{2} \frac{\partial^{2} \Psi}{\partial y^{2}}(x, y)-\frac{s^{2}}{1-M^{2}} \Psi(x, y)\right]=0 .
\end{gathered}
$$

Lemma 2.2. Let

$$
H_{0}^{(1)}=J_{0}+i Y_{0}
$$

be the Hankel function of the first kind of order 0 .

Then for fixed $\xi$ and $\eta$ function

$$
\Psi_{\xi, \eta}(x, y)=\frac{\partial}{\partial \eta} H_{0}^{(1)}(z)
$$

with

$$
z=\frac{i s}{a \sqrt{1-M^{2}}} \sqrt{\frac{(x-\xi)^{2}}{1-M^{2}}+(y-\eta)^{2}}
$$

satisfies equation (7).

Proof. Because of linearity of equation (7) it suffices to prove that function $H_{0}^{(1)}(z)$ satisfies this equation. For this function we have

$$
\begin{gathered}
a^{2}\left(1-M^{2}\right) \frac{\partial^{2} H_{0}^{(1)}(z)}{\partial x^{2}}+a^{2} \frac{\partial^{2} H_{0}^{(1)}(z)}{\partial y^{2}}-\frac{s^{2}}{1-M^{2}} H_{0}^{(1)}(z) \\
=a^{2}\left(1-M^{2}\right) \frac{\partial}{\partial x}\left[\frac{d H_{0}^{(1)}}{d z}(z) \frac{\partial z}{\partial x}\right]+a^{2} \frac{\partial}{\partial y}\left[\frac{d H_{0}^{(1)}}{d z}(z) \frac{\partial z}{\partial y}\right]-\frac{s^{2}}{1-M^{2}} H_{0}^{(1)}(z) \\
=-a^{2}\left(1-M^{2}\right) \frac{s^{2}}{a^{2}\left(1-M^{2}\right)} \frac{d^{2} H_{0}^{(1)}}{d z^{2}}(z) \cdot\left[\left(\sqrt{\frac{(x-\xi)^{2}}{1-M^{2}}+(y-\eta)^{2}}\right)^{-1} \cdot \frac{x-\xi}{1-M^{2}}\right]^{2} \\
+\frac{i s a^{2}\left(1-M^{2}\right)}{a \sqrt{1-M^{2}}} \frac{d H_{0}^{(1)}}{d z}(z) \cdot\left[\frac{1}{1-M^{2}}\left(\sqrt{\frac{(x-\xi)^{2}}{1-M^{2}}+(y-\eta)^{2}}\right)^{-1}\right.
\end{gathered}
$$




$$
\begin{aligned}
& \left.-\left(\frac{x-\xi}{1-M^{2}}\right)^{2}\left(\sqrt{\frac{(x-\xi)^{2}}{1-M^{2}}+(y-\eta)^{2}}\right)^{-3}\right] \\
& -a^{2} \frac{s^{2}}{a^{2}\left(1-M^{2}\right)} \frac{d^{2} H_{0}^{(1)}}{d z^{2}}(z) \cdot\left[\left(\sqrt{\frac{(x-\xi)^{2}}{1-M^{2}}+(y-\eta)^{2}}\right)^{-1} \cdot(y-\eta)\right]^{2} \\
& +a^{2} \frac{i s}{a \sqrt{1-M^{2}}} \frac{d H_{0}^{(1)}}{d z}(z) \cdot\left[\left(\sqrt{\frac{(x-\xi)^{2}}{1-M^{2}}+(y-\eta)^{2}}\right)^{-1}\right. \\
& \left.-\left(\sqrt{\frac{(x-\xi)^{2}}{1-M^{2}}+(y-\eta)^{2}}\right)^{-3} \cdot(y-\eta)^{2}\right] \\
& -\frac{s^{2}}{1-M^{2}} H_{0}^{(1)}(z) \\
& =-\frac{s^{2}}{1-M^{2}} \frac{d^{2} H_{0}^{(1)}}{d z^{2}}(z)\left[\left(\frac{(x-\xi)^{2}}{1-M^{2}}+(y-\eta)^{2}\right)^{-1} \cdot\left(\frac{(x-\xi)^{2}}{1-M^{2}}+(y-\eta)^{2}\right)\right] \\
& +2 \frac{i s a}{\sqrt{1-M^{2}}} \frac{d H_{0}^{(1)}}{d z}(z) \cdot\left(\sqrt{\frac{(x-\xi)^{2}}{1-M^{2}}+(y-\eta)^{2}}\right)^{-1} \\
& -\frac{i s a}{\sqrt{1-M^{2}}} \frac{d H_{0}^{(1)}}{d z}(z) \cdot\left(\sqrt{\frac{(x-\xi)^{2}}{1-M^{2}}+(y-\eta)^{2}}\right)^{-1}-\frac{s^{2}}{1-M^{2}} H_{0}^{(1)}(z) \\
& =\frac{-s^{2}}{1-M^{2}}\left[\frac{d^{2} H_{0}^{(1)}}{d z^{2}}(z)+\frac{d H_{0}^{(1)}}{d z}(z)\left(\frac{i s}{a \sqrt{1-M^{2}}} \sqrt{\frac{(x-\xi)^{2}}{1-M^{2}}+(y-\eta)^{2}}\right)^{-1}+H_{0}^{(1)}(z)\right]=0,
\end{aligned}
$$

where in the last equality we used the fact that function $H_{0}^{(1)}$ satisfies the Bessel equation of order zero.

From Lemma 2.2 we obtain that for arbitrary $\xi \in[-1,1]$ function

$$
\Psi_{\xi, 0}(x, y)=\frac{\partial}{\partial \eta} H_{0}^{(1)}(z)
$$

with

$$
z=\frac{i s}{a \sqrt{1-M^{2}}} \sqrt{\frac{(x-\xi)^{2}}{1-M^{2}}+y^{2}}
$$

is well defined in $\mathbb{R}^{2} \backslash[-1,1]$ and represents a solution of equation (7). In addition, formula

$$
\frac{\partial}{\partial \eta} H_{0}^{(1)}(z)=\frac{d H_{0}^{(1)}}{d z}(z) \cdot \frac{\partial z}{\partial \eta}=\frac{d H_{0}^{(1)}}{d z}(z) \frac{-i s(y-\eta)}{a \sqrt{1-M^{2}}} \cdot\left(\sqrt{\frac{(x-\xi)^{2}}{1-M^{2}}+(y-\eta)^{2}}\right)^{-1}
$$

shows that

$$
\Psi_{\xi, 0}(x, y)=0 \text { for }\{|x|>1, y=0\} .
$$

Therefore, by Lemma 2.1 function

$$
\psi_{\xi, 0}(x, y, t)=\Psi_{\xi, 0}(x, y) e^{s(t+c x)},
$$


with $c$ defined in formula (9), is a solution of equation (1) for arbitrary $s$. Following the spirit of terminology in $[\mathrm{BAH}]$ we call this solution a $(\xi, s)$-doublet and construct a general doublet-solution of equation (1) by the formula

$$
\psi(x, y, t)=\frac{1}{2 \pi i} \int_{\sigma-i \infty}^{\sigma+i \infty} d s e^{s(t+c x)} \int_{-1}^{1} d \xi p(\xi, s)\left(\left.\frac{\partial H_{0}^{(1)}}{\partial \eta}(z)\right|_{\eta=0}\right)
$$

with arbitrary function $p(\xi, s)$, satisfying condition

$$
\|p(\xi, \sigma+i \nu)\|_{L_{\nu}^{1}}<K
$$

uniformly with respect to $\xi$ and $\sigma \in\left[\sigma_{1}, \sigma_{2}\right]$.

\section{Generalized Possio integral equation.}

In this section we construct the velocity potential corresponding to a doublet-solution in (13) and represent the flow tangency condition (2) as an integral equation with respect to $p(\xi, s)$, which we call generalized Possio integral equation. Since condition (12) is satisfied by any $\Psi_{\xi, 0}$, condition (3) is automatically satisfied by arbitrary doublet-solution of the form (13). Therefore, in order to solve the boundary value problem under consideration we only need the corresponding velocity potential to satisfy condition (2).

We start the construction of the velocity potential with the construction of such a potential for a $(\xi, s)$-doublet. Considering the definition of $\psi$

$$
\psi=\frac{\partial \phi}{\partial t}+U \frac{\partial \phi}{\partial x}
$$

as a differential equation with respect to $\phi$ and assuming that

$$
\phi_{\xi, 0}(x, y, t)=\Phi_{\xi, 0}(x, y) e^{s(t+c x)},
$$

we obtain an equation for $\Phi_{\xi, 0}$

$$
\frac{\partial \phi_{\xi, 0}}{\partial t}+U \frac{\partial \phi_{\xi, 0}}{\partial x}=s \Phi e^{s(t+c x)}+U s c \Phi e^{s(t+c x)}+U \frac{\partial \Phi}{\partial x} e^{s(t+c x)}=\left.\frac{\partial}{\partial \eta} H_{0}^{(1)}(z)\right|_{\eta=0} e^{s(t+c x)}
$$

or

$$
U \frac{\partial \Phi}{\partial x}+s(1+c U) \Phi=\left.\frac{\partial}{\partial \eta} H_{0}^{(1)}(z)\right|_{\eta=0}
$$

Solving this equation we obtain

$$
\Phi_{\xi, 0}(x, y)=\frac{e^{\lambda x}}{U} \int_{-\infty}^{x} e^{-\lambda u}\left(\left.\frac{\partial}{\partial \eta} H_{0}^{(1)}(\zeta)\right|_{\eta=0}\right) d u
$$

with

$$
\lambda=\lambda(s)=-\frac{s(1+c U)}{U},
$$

and

$$
\zeta=\frac{i s}{a \sqrt{1-M^{2}}} \sqrt{\frac{(u-\xi)^{2}}{1-M^{2}}+(y-\eta)^{2}} .
$$

Finally, from (14) using equality

$$
\left.\frac{\partial H_{0}^{(1)}}{\partial \eta}(z)\right|_{\eta=0}=\left.\frac{d H_{0}^{(1)}}{d z} \cdot \frac{\partial z}{\partial \eta}\right|_{\eta=0}=-\left.\frac{\partial H_{0}^{(1)}}{\partial y}(z)\right|_{\eta=0} .
$$


we obtain an expression for the velocity potential corresponding to arbitrary doubletsolution

$$
\phi(x, y, t)=-\frac{1}{2 \pi i} \int_{\sigma-i \infty}^{\sigma+i \infty} d s e^{s(t+c x)} \frac{e^{\lambda(s) x}}{U} \int_{-1}^{1} p(\xi, s) d \xi \int_{-\infty}^{x} e^{-\lambda(s) u}\left(\left.\frac{\partial H_{0}^{(1)}}{\partial y}(\zeta)\right|_{\eta=0}\right) d u .
$$

Using formula (16) we rewrite the flow tangency condition

$$
\lim _{y \rightarrow 0^{+}} \frac{\partial \phi}{\partial y}(x, y, t)=w(x, t) \text { for } x \in[-1,1]
$$

as

$$
=-\frac{1}{2 \pi i} \lim _{y \rightarrow 0^{+}} \int_{\sigma-i \infty}^{\sigma+i \infty} d s e^{s(t+c x)} \frac{e^{\lambda(s) x}}{U} \int_{-1}^{1} p(\xi, s) d \xi \int_{-\infty}^{x} e^{-\lambda(s) u}\left(\left.\frac{\partial^{2} H_{0}^{(1)}}{\partial y^{2}}(\zeta)\right|_{\eta=0}\right) d u .
$$

Representing then

$$
w(x, t)=\frac{1}{2 \pi i} \int_{\sigma-i \infty}^{\sigma+i \infty} e^{s t} \widehat{w}(x, s) d s
$$

we transform the equation above into

$$
\begin{aligned}
\frac{1}{2 \pi i} \int_{\sigma-i \infty}^{\sigma+i \infty} d s e^{s t}\left\{\widehat{w}(x, s)+\lim _{y \rightarrow 0^{+}} e^{s c x} \frac{e^{\lambda(s) x}}{U} \int_{-1}^{1} p(\xi, s) d \xi \int_{-\infty}^{x} e^{-\lambda(s) u}\right. \\
\left.\times\left(\left.\frac{\partial^{2} H_{0}^{(1)}}{\partial y^{2}}(\zeta)\right|_{\eta=0}\right) d u\right\}=0
\end{aligned}
$$

and further into

$$
\widehat{w}(x, s) e^{-s c x}=-\lim _{y \rightarrow 0^{+}} \frac{e^{\lambda(s) x}}{U} \int_{-1}^{1} d \xi p(\xi, s) \int_{-\infty}^{x} d u e^{-\lambda(s) u}\left(\left.\frac{\partial^{2} H_{0}^{(1)}}{\partial y^{2}}(\zeta)\right|_{\eta=0}\right) .
$$

To transform equation above into a singular integral equation we follow two additional steps from the Possio's scheme $([\mathrm{BAH}])$. On the first step we use the fact that $H_{0}^{(1)}(z)$ satisfies equation (7), and therefore

$$
\frac{\partial^{2} H_{0}^{(1)}}{\partial y^{2}}(z)=-\left(1-M^{2}\right) \frac{\partial^{2} H_{0}^{(1)}}{\partial x^{2}}(z)+\frac{s^{2}}{a^{2}\left(1-M^{2}\right)} H_{0}^{(1)}(z) .
$$

Then we obtain equation

$$
\begin{gathered}
\widehat{w}(x, s) e^{-s c x}=\lim _{y \rightarrow 0^{+}} \frac{e^{\lambda(s) x}}{U} \int_{-1}^{1} d \xi p(\xi, s) \\
\times\left.\int_{-\infty}^{x} d u e^{-\lambda(s) u}\left(\left(1-M^{2}\right) \frac{\partial^{2} H_{0}^{(1)}}{\partial u^{2}}(\zeta)-\frac{s^{2}}{a^{2}\left(1-M^{2}\right)} H_{0}^{(1)}(\zeta)\right)\right|_{\eta=0} .
\end{gathered}
$$

On the second step we transform the second integral in the equation above using integration by parts in the formula

$$
\begin{gathered}
\int_{-\infty}^{x} e^{-\lambda u} \frac{\partial^{2} H_{0}^{(1)}}{\partial u^{2}}(\zeta) d u=\left.e^{-\lambda u} \cdot \frac{\partial H_{0}^{(1)}}{\partial u}(\zeta)\right|_{-\infty} ^{x}+\lambda \int_{-\infty}^{x} e^{-\lambda u} \frac{\partial H_{0}^{(1)}}{\partial u}(\zeta) d u \\
=e^{-\lambda x} \cdot \frac{\partial H_{0}^{(1)}}{\partial x}(z)+\left.\lambda e^{-\lambda u} \cdot H_{0}^{(1)}(\zeta)\right|_{-\infty} ^{x}+\lambda^{2} \int_{-\infty}^{x} e^{-\lambda u} H_{0}^{(1)}(\zeta) d u
\end{gathered}
$$




$$
=e^{-\lambda x} \cdot \frac{\partial H_{0}^{(1)}}{\partial x}(z)+\lambda e^{-\lambda x} \cdot H_{0}^{(1)}(z)+\lambda^{2} \int_{-\infty}^{x} e^{-\lambda u} H_{0}^{(1)}(\zeta) d u .
$$

Using formula (17) in the equation above we obtain the generalized Possio integral equation

$$
\begin{gathered}
\widehat{w}(x, s) e^{-s c x}=\int_{-1}^{1} d \xi p(\xi, s) \lim _{y \rightarrow 0^{+}}\left[\frac{\left(1-M^{2}\right)}{U} \cdot \frac{\partial H_{0}^{(1)}}{\partial x}(z)+\frac{\lambda(s)\left(1-M^{2}\right)}{U} H_{0}^{(1)}(z)\right. \\
\left.+\frac{e^{\lambda(s) x}}{U}\left(\lambda^{2}(s)\left(1-M^{2}\right)-\frac{s^{2}}{a^{2}\left(1-M^{2}\right)}\right) \int_{-\infty}^{x} e^{-\lambda(s) u} H_{0}^{(1)}(\zeta) d u\right]\left.\right|_{\eta=0} .
\end{gathered}
$$

This equation reduces to the Possio integral equation ([BAH] $)$ if we fix $s$ and consider oscillations with fixed frequency. In this case function $w(x, t)$ reduces to $w(x) e^{i k t}$ and the sought solution $p(\xi, s)$ reduces to $p(\xi)$.

\section{Finite Hilbert transform.}

Before analyzing the solvability of the integral equation (18) we represent this equation as an integral equation with the principal term being a multiple of the finite Hilbert transform [Tr]. In order to obtain such a representation we use Taylor (respectively Laurent) series of Bessel functions $Y_{0}$ and $Y_{1}$ ([EMOT], v.II, 7.2.4, (32)).

Proposition 4.1. Kernel of the generalized Possio equation (18) admits the following representation

$$
\begin{gathered}
\lim _{y \rightarrow 0^{+}}\left[\frac{\left(1-M^{2}\right)}{U} \cdot \frac{\partial H_{0}^{(1)}}{\partial x}(z)+\frac{\lambda(s)\left(1-M^{2}\right)}{U} H_{0}^{(1)}(z)\right. \\
\left.+\frac{e^{\lambda(s) x}}{U}\left(\lambda^{2}(s)\left(1-M^{2}\right)-\frac{s^{2}}{a^{2}\left(1-M^{2}\right)}\right) \int_{-\infty}^{x} e^{-\lambda(s) u} H_{0}^{(1)}(\zeta) d u\right]\left.\right|_{\eta=0} \\
=-\frac{2 i\left(1-M^{2}\right)^{3 / 2}}{\pi U} \frac{1}{(x-\xi)}+K(x, \xi, s)
\end{gathered}
$$

where

$$
K(x, \xi, s)=A(x, \xi, s) \log |x-\xi|+B(x, \xi, s),
$$

with functions $A$ and $B$ bounded with respect to $x, \xi$, analytically depending on $s$ and satisfying estimate

$$
\sup _{x, \xi \in[-1,1]}\left\{\left|A_{1}(x, \xi, s)\right|,\left|B_{1}(x, \xi, s)\right|\right\}<C|s|^{2} .
$$

Proof. We consider the first term of the kernel of equation (18), for which using equality

$$
\frac{d}{d z} H_{0}^{(1)}(z)=-H_{1}^{(1)}(z)
$$

we obtain

$$
\begin{gathered}
\left.\frac{1-M^{2}}{U} \lim _{y \rightarrow 0^{+}}\left(\frac{\partial H_{0}^{(1)}(z)}{\partial x}\right)\right|_{\eta=0}=\left.\frac{1-M^{2}}{U} \lim _{y \rightarrow 0^{+}} \frac{d H_{0}^{(1)}}{d z}(z) \cdot \frac{\partial z}{\partial \eta}\right|_{\eta=0} \\
=-\frac{1-M^{2}}{U} H_{1}^{(1)}\left(\frac{i s|x-\xi|}{a\left(1-M^{2}\right)}\right) \cdot \frac{i s(x-\xi)}{a|x-\xi| \sqrt{1-M^{2}}}
\end{gathered}
$$




$$
=-\frac{1-M^{2}}{U}\left[J_{1}\left(\frac{i s|x-\xi|}{a\left(1-M^{2}\right)}\right)+i Y_{1}\left(\frac{i s|x-\xi|}{a\left(1-M^{2}\right)}\right)\right] \cdot \frac{i s(x-\xi)}{a|x-\xi| \sqrt{1-M^{2}}},
$$

where $J_{1}$ and $Y_{1}$ are Bessel functions.

For the case $|s(x-\xi)|<C$ with $\operatorname{Re} s \in\left[\sigma_{1}, \sigma_{2}\right]$ we use the Laurent series of $Y_{1}$, analyticity of $J_{1}$ and boundedness of

$$
\frac{i s(x-\xi)}{a|x-\xi| \sqrt{1-M^{2}}}
$$

and obtain the following formula

$$
\begin{gathered}
\left.\frac{1-M^{2}}{U} \lim _{y \rightarrow 0^{+}}\left(\frac{\partial H_{0}^{(1)}(z)}{\partial x}\right)\right|_{\eta=0} \\
=-\frac{2 i\left(1-M^{2}\right)^{3 / 2}}{\pi U} \frac{1}{(x-\xi)}+A_{1}(x, \xi, s) \log |x-\xi|+B_{1}(x, \xi, s)
\end{gathered}
$$

with functions $A_{1}$ and $B_{1}$ bounded with respect to $x, \xi$ for fixed $s$, analytically depending on $s$, and satisfying

$$
\sup _{x, \xi \in[-1,1]}\left\{\left|A_{1}(x, \xi, s)\right|,\left|B_{1}(x, \xi, s)\right|\right\}<C|s|
$$

for some $C>0$.

For the case $|s(x-\xi)|>C$ with $\operatorname{Re} s \in\left[\sigma_{1}, \sigma_{2}\right]$ we again use formula (22) and asymptotic expansions of Hankel functions for large $|z|$ ([EMOT], v.II, 7.13). Then we obtain representation

$$
\left.\frac{1-M^{2}}{U} \lim _{y \rightarrow 0^{+}}\left(\frac{\partial H_{0}^{(1)}(z)}{\partial x}\right)\right|_{\eta=0}=A_{2}(x, \xi, s) \log |x-\xi|+B_{2}(x, \xi, s)
$$

with functions $A_{2}$ and $B_{2}$ bounded with respect to $x, \xi$ for fixed $s$, analytically depending on $s$, and satisfying estimate (24).

For the rest of the kernel of equation (18) in the case $|s(x-\xi)|<C$ with $\operatorname{Re} s \in\left[\sigma_{1}, \sigma_{2}\right]$ we use the Taylor series of $Y_{0}$ and obtain

$$
\begin{gathered}
\lim _{y \rightarrow 0^{+}}\left[\frac{\lambda(s)\left(1-M^{2}\right)}{U} H_{0}^{(1)}(z)\right. \\
\left.+\frac{e^{\lambda(s) x}}{U}\left(\lambda^{2}(s)\left(1-M^{2}\right)-\frac{s^{2}}{a^{2}\left(1-M^{2}\right)}\right) \int_{-\infty}^{x} e^{-\lambda(s) u} H_{0}^{(1)}(\zeta) d u\right]\left.\right|_{\eta=0} \\
=A_{3}(x, \xi, s) \log |x-\xi|+B_{3}(x, \xi, s)
\end{gathered}
$$

with functions $A_{3}(x, \xi, s)$ and $B_{3}(x, \xi, s)$ analytically depending on $s$ and satisfying estimate (21).

In the case $|s(x-\xi)|>C$ with Res $\in\left[\sigma_{1}, \sigma_{2}\right]$ we again use asymptotic expansions of Hankel functions for large $|z|$. Then we obtain representation, similar to (26) with functions satisfying estimate (21).

Combining formulas above we obtain representation (19) of the kernel of the generalized Possio equation with functions $A(x, \xi, s)$ and $B(x, \xi, s)$ satisfying estimate $(21)$. 


\section{Solvability of the generalized Possio equation.}

Using representation (19) we consider operators

$$
\mathcal{K}_{s}[f](x)=\int_{-1}^{1} f(\xi) K(x, \xi, s) d \xi
$$

and

$$
\mathcal{R}_{s}[f](x)=\mathcal{T}[f](x)-\frac{i U}{2\left(1-M^{2}\right)^{3 / 2}} \mathcal{K}_{s}[f](x),
$$

where $\mathcal{T}$ is the finite Hilbert transform [Tr]:

$$
\mathcal{T}[f](x)=\frac{1}{\pi} \int_{-1}^{1} \frac{f(\xi)}{\xi-x} d \xi .
$$

Then we can rewrite equation (18) in the following form

$$
\mathcal{R}_{s}[p](x)=-\frac{i U}{2\left(1-M^{2}\right)^{3 / 2}} \widehat{w}(x, s) e^{-s c x},
$$

with unknown function $p(\xi, s)$.

An important role in the analysis of solvability of equation (18) plays operator described in the following proposition ([So], $[\mathrm{Tr}])$.

Proposition 5.1. Operator $\mathcal{T}^{-1}$ defined by the formula

$$
\mathcal{T}^{-1}[g](x)=-\frac{1}{\pi} \int_{-1}^{1} \sqrt{\frac{1-y^{2}}{1-x^{2}}} \frac{g(y)}{y-x} d y,
$$

is a bounded linear operator from $L^{\frac{4}{3}+}[-1,1]$ into $L^{p}[-1,1]$ for any $p<\frac{4}{3}$, satisfying equality

$$
\mathcal{T} \circ \mathcal{T}^{-1}[f]=f
$$

Using operator $\mathcal{T}^{-1}$ we reduce solution of equation (27), and therefore of equation (18), to the solution of equation

where

$$
\mathcal{G}_{s}[r](x)=-\frac{i U}{2\left(1-M^{2}\right)^{3 / 2}} \widehat{w}(x, s) e^{-s c x},
$$

$$
\mathcal{G}_{s}=\mathcal{R}_{s} \circ \mathcal{T}^{-1}=\mathcal{I}+\mathcal{N}_{s}
$$

with $\mathcal{I}$ - the identity operator and

$$
\mathcal{N}_{s}=-\frac{i U}{2\left(1-M^{2}\right)^{3 / 2}} \mathcal{K}_{s} \circ \mathcal{T}^{-1}
$$

The advantage of equation (29) over equation (27) becomes clear from the proposition below, in which we prove the Fredholm property of the family of operators $\mathcal{G}_{s}$. This proposition is the key new ingredient in the analysis of solvability of the Possio integral equation and is inspired by the Proposition 5.1 from $[\mathrm{P}]$.

Proposition 5.2. For any fixed $s \in \mathbb{C}$ operator $\mathcal{N}_{s}$ is compact on $L^{2}[-1,1]$, and therefore operator $\mathcal{G}_{s}$ defined in (30) is a Fredholm operator on $L^{2}[-1,1]$. In addition, kernel $N(x, y, \lambda)$ of the operator $\mathcal{N}_{s}$ admits estimate

$$
\int_{\mathbb{R}^{2}}|N(x, y, s)|^{2} d x d y<C|s|^{4}
$$

with constant $C$ independent of $s$. 
Proof. Using formula (28) for $\mathcal{T}^{-1}$ we obtain

$$
\begin{gathered}
\mathcal{N}_{s}[f](x)=-\frac{i U}{2\left(1-M^{2}\right)^{3 / 2}} \mathcal{K}_{s}\left[-\frac{1}{\pi} \int_{-1}^{1} \sqrt{\frac{1-y^{2}}{1-u^{2}}} \frac{f(y)}{y-u} d y\right] \\
=\frac{i U}{2\left(1-M^{2}\right)^{3 / 2}} \int_{-1}^{1} d u K(x, u, s) \int_{-1}^{1} \sqrt{\frac{1-y^{2}}{1-u^{2}}} \frac{f(y)}{y-u} d y \\
=\int_{-1}^{1} N(x, y, s) f(y) d y
\end{gathered}
$$

where

$$
N(x, y, s)=\frac{i U}{2\left(1-M^{2}\right)^{3 / 2}} \int_{-1}^{1} d u \frac{K(x, u, s)}{y-u} \sqrt{\frac{1-y^{2}}{1-u^{2}}} .
$$

To prove compactness of the operator $\mathcal{N}_{s}$ we use representation

$$
N(x, y, s)=\frac{i U}{2\left(1-M^{2}\right)^{3 / 2}}\left[N_{1}(x, y, s)+N_{2}(x, y, s)\right]
$$

with

and

$$
N_{1}(x, y, s)=\int_{-1}^{1} K(x, u, s) \frac{d u}{y-u}
$$

$$
\begin{gathered}
N_{2}(x, y, s)=\int_{-1}^{1} K(x, u, s) \frac{\left(\sqrt{1-y^{2}}-\sqrt{1-u^{2}}\right)}{\sqrt{1-u^{2}}} \frac{d u}{y-u} \\
=-\int_{-1}^{1} K(x, u, s) \frac{(y+u) d u}{\left(\sqrt{1-y^{2}}+\sqrt{1-u^{2}}\right) \sqrt{1-u^{2}}},
\end{gathered}
$$

and prove Hilbert-Schmidt property (cf.[L]) of kernels $N_{1}(x, y, s)$ and $N_{2}(x, y, s)$.

For $N_{1}$ we notice that according to estimates (21) for fixed $x \in[-1,1]$

$$
\int_{-1}^{1} K(x, u, s) \frac{d u}{y-u}
$$

is a multiple of the Hilbert transform of an $L^{2}[-1,1]$ - function $K(x, u, s)$ satisfying

$$
\|K(x, u, s)\|_{L_{u}^{2}}<C|s|^{2}
$$

with constant $C$ independent of $x$. Therefore,

$$
\begin{gathered}
\int_{-1}^{1} d x \int_{-1}^{1} d y\left|N_{1}(x, y, s)\right|^{2}=\int_{-1}^{1} d x \int_{-1}^{1} d y\left|\int_{-1}^{1} K(x, u, s) \frac{d u}{y-u}\right|^{2} \\
<C \int_{-1}^{1} d x\|K(x, u, s)\|_{L^{2}[-1,1]_{u}}^{2}<C|s|^{4} .
\end{gathered}
$$

For $N_{2}(x, y, s)$ we have

$$
\begin{aligned}
& \int_{-1}^{1} d x \int_{-1}^{1} d y\left|N_{2}(x, y, s)\right|^{2} \\
& =\int_{-1}^{1} d x \int_{-1}^{1} d y\left|\int_{-1}^{1} K(x, u, s) \frac{(y+u) d u}{\left(\sqrt{1-y^{2}}+\sqrt{1-u^{2}}\right) \sqrt{1-u^{2}}}\right|^{2} \\
& <C|s|^{4} \int_{-1}^{1} d x \int_{-1}^{1} d y\left|\int_{-1}^{1} \frac{\log |x-u| d u}{\left(\sqrt{1-y^{2}}+\sqrt{1-u^{2}}\right) \sqrt{1-u^{2}}}\right|^{2}
\end{aligned}
$$


where in the last inequality we used representation (20) of function $K$.

To estimate the last integral in (32) we define

$$
S_{x, y}=\left\{u:|x-u| \geq \frac{1}{2}|x-1| \cdot \sqrt{1-y^{2}}\right\}
$$

and estimate separately integrals over $S_{x, y}$ and $[-1,1] \backslash S_{x, y}$. For the integral over $S_{x, y}$, changing variable $u=\cos \theta$, we have

$$
\begin{gathered}
\int_{-1}^{1} d x \int_{-1}^{1} d y\left|\int_{-1}^{1} \frac{\log |x-u| d u}{\left(\sqrt{1-y^{2}}+\sqrt{1-u^{2}}\right) \sqrt{1-u^{2}}}\right|^{2} \\
<C \int_{-1}^{1} d x \int_{-1}^{1} d y\left|\int_{0}^{\pi} \frac{\left(\log |1-x|+\log \sqrt{1-y^{2}}\right) d \theta}{\left(\sqrt{1-y^{2}}+|\sin \theta|\right)}\right|^{2} \\
<C \int_{-1}^{1} d x \int_{-1}^{1} d y\left(\log |1-x|+\log \sqrt{1-y^{2}}\right)^{2}\left(\log \sqrt{1-y^{2}}\right)^{2}<C .
\end{gathered}
$$

For $u \in[0,1] \backslash S_{x, y}$ we have

$$
|1-u| \geq|1-x|-|x-u| \geq|1-x|-\frac{1}{2}|1-x| \cdot \sqrt{1-y^{2}} \geq \frac{1}{2}|1-x| .
$$

Therefore, for the integral over $[0,1] \backslash S_{x, y}$ we obtain

$$
\begin{gathered}
\int_{-1}^{1} d x \int_{-1}^{1} d y\left|\int_{[0,1] \backslash S_{x, y}} \frac{\log |x-u| d u}{\left(\sqrt{1-y^{2}}+\sqrt{1-u^{2}}\right) \sqrt{1-u^{2}}}\right|^{2} \\
<C \int_{-1}^{1} d x \int_{-1}^{1} d y\left|\frac{1}{\sqrt{1-y^{2}} \sqrt{|1-x|}} \int_{x-\frac{1}{2}|x-1| \cdot \sqrt{1-y^{2}}}^{x+\frac{1}{2}|x-1| \cdot \sqrt{1-y^{2}}} \log \right| x-u|d u|^{2} \\
<C \int_{-1}^{1} d x \int_{-1}^{1} d y\left|\frac{\left(|1-x| \cdot \sqrt{1-y^{2}}\right)\left(\log |1-x|+\log \sqrt{1-y^{2}}\right)}{\sqrt{1-y^{2}} \sqrt{|1-x|}}\right|^{2}<C .
\end{gathered}
$$

The same estimate holds for the integral over $[-1,0] \backslash S_{x, y}$.

Combining the estimates above we obtain estimate (31).

To formulate a criterion of solvability of equation (18) for a fixed $s \in \mathbb{C}$ we define a complex number $s_{0}$ to be a characteristic value of the family of operators $\mathcal{G}_{s}$ if operator $\mathcal{G}_{s_{0}}$ is not invertible in $L^{2}[-1,1]$. Using Propositions 5.1 and 5.2 we obtain the following criterion.

Proposition 5.3. If $s_{0}$ is not a characteristic value of the family of operators $\mathcal{G}_{s}$ and $\widehat{w}\left(x, s_{0}\right) e^{-s_{0} c x} \in L^{2}[-1,1]$, then there exists a function $p\left(\xi, s_{0}\right) \in L^{\frac{4}{3}-}[-1,1]$ satisfying equation (18).

Proof. We consider a solution $r$ of equation

$$
\mathcal{G}_{s_{0}}[r]=\mathcal{R}_{s_{0}} \circ \mathcal{T}^{-1}[r]=-\frac{i U}{2\left(1-M^{2}\right)^{3 / 2}} \widehat{w}\left(x, s_{0}\right) e^{-s_{0} c x} .
$$

Then we have for $p\left(\xi, s_{0}\right)=\mathcal{T}^{-1}[r]$

$$
\mathcal{R}_{s_{0}}[p]=\mathcal{R}_{s_{0}} \circ \mathcal{T}^{-1}[r]=-\frac{i U}{2\left(1-M^{2}\right)^{3 / 2}} \widehat{w}\left(x, s_{0}\right) e^{-s_{0} c x} .
$$




\section{The Resolvent of operator $\mathcal{G}_{s}$.}

In this section we address the question of solvability of integral equation (29) with varying $s$. We construct the resolvent of the operator $\mathcal{G}_{s}$ and show that the resolvent is also a Fredholm operator analytically depending on $s \in\left\{\operatorname{Re} s>\sigma_{1}\right\}$.

Let $\mathcal{P}: L^{2}(\mathbb{R}) \rightarrow L^{2}(\mathbb{R})$ be an integral operator with kernel $P(x, y)$ satisfying HilbertSchmidt condition. Following [C], we consider for operator $\mathcal{P}$ Hilbert's modification of the original Fredholm's determinants:

$$
\begin{gathered}
D_{P, m}\left(t_{1}, \ldots, t_{m}\right)=\left|\begin{array}{cccc}
0 & P\left(t_{1}, t_{2}\right) & \ldots & P\left(t_{1}, t_{m}\right) \\
P\left(t_{2}, t_{1}\right) & 0 & \ldots & P\left(t_{2}, t_{m}\right) \\
\vdots & & & \vdots \\
P\left(t_{m}, t_{1}\right) & \ldots & P\left(t_{m}, t_{m-1}\right) & 0
\end{array}\right|, \\
D_{P}=1+\sum_{m=1}^{\infty} \delta_{m}=1+\sum_{m=1}^{\infty} \frac{1}{m !} \int_{\mathbb{R}} \ldots \int_{\mathbb{R}} D_{P, m}\left(t_{1}, \ldots, t_{m}\right) d t_{1} \cdots d t_{m}, \\
D_{P, m}\left(\begin{array}{c}
x \\
y
\end{array} t_{1}, \ldots, t_{m}\right)=\left|\begin{array}{cccc}
P(x, y) & P\left(x, t_{1}\right) & \ldots & P\left(x, t_{m}\right) \\
P\left(t_{1}, y\right) & 0 & \ldots & P\left(t_{1}, t_{m}\right) \\
\vdots & & & \vdots \\
P\left(t_{m}, y\right) & \ldots & P\left(t_{m}, t_{m-1}\right) & 0
\end{array}\right|,
\end{gathered}
$$

and

$$
\begin{aligned}
& D_{P}\left(\begin{array}{l}
x \\
y
\end{array}\right)=P(x, y)+\sum_{m=1}^{\infty} \delta_{m}\left(\begin{array}{l}
x \\
y
\end{array}\right) \\
& =P(x, y)+\sum_{m=1}^{\infty} \frac{1}{m !} \int_{\mathbb{R}} \cdots \int_{\mathbb{R}} D_{P, m}\left(\begin{array}{l}
x \\
y
\end{array} t_{1}, \ldots, t_{m}\right) d t_{1} \cdots d t_{m} .
\end{aligned}
$$

We start with the following proposition, which summarizes the results from [C] (cf. also $[\mathrm{M}])$, that will be used in the construction of the resolvent of $\mathcal{G}_{s}$.

Proposition 6.1. ([C]) Let function $P(x, y): \mathbb{R}^{2} \rightarrow \mathbb{C}$ satisfy Hilbert-Schmidt condition

$$
\|P\|^{2}=\int_{\mathbb{R}^{2}}|P(x, y)|^{2} d x d y<\infty .
$$

Then function $D_{P}\left(\begin{array}{l}x \\ y\end{array}\right) \in L^{2}\left(\mathbb{R}^{2}\right)$ is well defined, and the following estimates hold:

$$
\begin{gathered}
\left|\delta_{m}\right| \leq\left(\frac{e}{m}\right)^{m / 2}\|P\|^{m},\left|D_{P}\right| \leq e^{\frac{\|P\|^{2}}{2}}, \\
\left|D_{P}\left(\begin{array}{c}
x \\
y
\end{array}\right)\right| \leq e^{\frac{\|P\|^{2}}{2}}(|P(x, y)|+\sqrt{e} \alpha(x) \beta(y)),
\end{gathered}
$$

where

$$
\alpha^{2}(x)=\int_{\mathbb{R}}|P(x, t)|^{2} d t, \quad \beta^{2}(y)=\int_{\mathbb{R}}|P(t, y)|^{2} d t .
$$

If $D_{P} \neq 0$ then kernel

$$
H(x, y)=\left[D_{P}\right]^{-1} \cdot D_{P}\left(\begin{array}{l}
x \\
y
\end{array}\right)
$$


defines the resolvent of operator $\mathcal{I}-\mathcal{P}$, i.e. it satisfies the following equations

$$
\begin{aligned}
& H(x, y)+\int_{\mathbb{R}} P(x, t) \cdot H(t, y) d t=P(x, y), \\
& H(x, y)+\int_{\mathbb{R}} P(t, y) \cdot H(x, t) d t=P(x, y),
\end{aligned}
$$

and therefore operator $\mathcal{I}-\mathcal{H}$ is the inverse of operator $\mathcal{I}+\mathcal{P}$.

In the next proposition we obtain necessary estimate for the resolvent $\mathcal{I}-\mathcal{H}_{s}$ with respect to $s$.

Proposition 6.2. ([P]) The set of characteristic values of the family of operators $\mathcal{G}_{s}$ coincides with the set

$$
E(\mathcal{G})=\left\{s \in \mathbb{C}: \text { Res }>\sigma_{1}, D_{N_{s}}=0\right\}
$$

and consists of at most countably many isolated points.

For $s \notin E(\mathcal{G})$ there exists an operator $\mathcal{H}_{s}$ with kernel $H(x, y, s)$ satisfying the HilbertSchmidt condition and such that operator $\mathcal{I}-\mathcal{H}_{s}$ is the inverse of operator $\mathcal{I}+\mathcal{N}_{s}=\mathcal{G}_{s}$.

If function $D_{N}(s)=D_{N_{s}}$ has no zeros in a strip $\left\{s=\sigma+i \nu: \sigma_{1}<\right.$ Res $\left.<\sigma_{2}\right\}$, then operator $\mathcal{H}_{s}$ admits estimate

$$
\left\|\mathcal{H}_{s}\right\|<\exp \left\{C e^{|\nu|} \cdot(1+|\nu|)^{4}\right\}
$$

for some $C>0, s \in\left\{\sigma_{1}+\gamma<\right.$ Res $\left.<\sigma_{2}-\gamma\right\}$, and arbitrary $\epsilon>0$.

Proof. This proposition is a copy of Proposition 6.2 from $[\mathrm{P}]$ except estimate (39). As in that proposition, analyticity of $\mathcal{H}_{s}$ and countability of the set of characteristic values follow from Proposition 6.1 and Theorem VI.14 from [RS] applied to the family of operators $\mathcal{N}_{s}$.

Estimate (39) is proved by applying the standard estimate

$$
\|\mathcal{P}\|^{2} \leq \int_{\mathbb{R}^{2}}|P(x, y)|^{2} d x d y
$$

for an integral operator $\mathcal{P}$ with kernel $P(x, y)$, estimate from the Lemma 6.3 below and the following estimate

$$
\left\|D_{N}\left(\begin{array}{c}
x \\
y
\end{array} \mid s\right)\right\|<\exp \left\{C(1+|\nu|)^{4}\right\} \cdot(1+|\nu|)^{4} \text { for } s=\sigma+i \nu .
$$

To obtain estimate (40) we use estimate (36). Then we have

$$
\begin{gathered}
\left\|D_{N}\left(\begin{array}{c}
x \\
y
\end{array} \mid s\right)\right\|^{2}<C e^{\left\|\mathcal{N}_{s}\right\|^{2}} \cdot \int_{\mathbb{R}^{2}} d x d y\left[\left|N_{s}(x, y)\right|^{2}+\alpha^{2}(x) \beta^{2}(y)\right] \\
=C e^{\left\|\mathcal{N}_{s}\right\|^{2}} \cdot\left[\int_{\mathbb{R}^{2}}\left|N_{s}(x, y)\right|^{2} d x d y+\int_{\mathbb{R}} \alpha^{2}(x) d x \cdot \int_{\mathbb{R}} \beta^{2}(y) d y\right] \\
=C e^{\left\|\mathcal{N}_{s}\right\|^{2}} \cdot\left[\int_{\mathbb{R}^{2}}\left|N_{s}(x, y)\right|^{2} d x d y+\int_{\mathbb{R}} d x \int_{\mathbb{R}} d t\left|N_{s}(x, t)\right|^{2} \cdot \int_{\mathbb{R}} d y \int_{\mathbb{R}} d t\left|N_{s}(t, y)\right|^{2}\right] \\
<C \exp \left\{|s|^{4}\right\} \cdot\left(|s|^{4}+|s|^{8}\right)
\end{gathered}
$$

where in the last inequality we used estimate (31).

Finally, in order to use formula (37) for the estimate of $\left\|\mathcal{H}_{s}\right\|$ we need an estimate of the function $\left|1 / D_{N}(s)\right|$, which is given in the lemma below. 
Lemma 6.3. ([P]) If function $D_{N}(s)=D_{N_{s}}$ has no zeros in the strip $\left\{s: \sigma_{1}<\right.$ Res $\left.<\sigma_{2}\right\}$, then estimate

$$
\left|1 / D_{N}(s)\right|<\exp \left\{C e^{|\nu|} \cdot(1+|\nu|)^{4}\right\}
$$

holds for $s=\sigma+i \nu \in\left\{\sigma_{1}+\gamma<\right.$ Res $\left.<\sigma_{2}-\gamma\right\}$ with fixed $\gamma>0$ and arbitrary $\epsilon>0$.

Proof. We consider a biholomorphic map

$$
\Psi:\left\{s: \sigma_{1}<\operatorname{Re} s<\sigma_{2}\right\} \rightarrow \mathbb{D}(1)=\{z \in \mathbb{C}:|z|<1\},
$$

defined by the formula

Denoting

$$
\Psi(s)=\frac{e^{i\left(s-\sigma_{1}\right) \frac{\pi}{\sigma_{2}-\sigma_{1}}}-i}{e^{i\left(s-\sigma_{1}\right) \frac{\pi}{\sigma_{2}-\sigma_{1}}}+i} .
$$

$$
w=u+i v=e^{i\left(s-\sigma_{1}\right) \frac{\pi}{\sigma_{2}-\sigma_{1}}},
$$

we obtain for the circle $C(r)=\{z:|z|=r\}$

$$
\begin{gathered}
\Psi^{-1}(C(r))=\left\{\sigma+i \nu:\left|e^{i\left(s-\sigma_{1}\right) \frac{\pi}{\sigma_{2}-\sigma_{1}}}-i\right|=r\left|e^{i\left(s-\sigma_{1}\right) \frac{\pi}{\sigma_{2}-\sigma_{1}}}+i\right|\right\} \\
=\left\{u+i v:\left(u^{2}+v^{2}-2 v+1\right)=r^{2}\left(u^{2}+v^{2}+2 v+1\right)\right\} \\
=\left\{u+i v: u^{2}+\left(v-\frac{1+r^{2}}{1-r^{2}}\right)^{2}=\frac{4 r^{2}}{\left(1-r^{2}\right)^{2}}\right\} .
\end{gathered}
$$

Introducing coordinates

such that

$$
\rho=\operatorname{Re} \frac{\pi\left(s-\sigma_{1}\right)}{\sigma_{2}-\sigma_{1}}, \tau=\operatorname{Im} \frac{\pi\left(s-\sigma_{1}\right)}{\sigma_{2}-\sigma_{1}},
$$

$$
w=u+i v=e^{i\left(s-\sigma_{1}\right) \frac{\pi}{\sigma_{2}-\sigma_{1}}}=e^{i \rho-\tau}=e^{-\tau}(\cos \rho+\sin \rho),
$$

we can rewrite the last condition as a quadratic equation with respect to $e^{-\tau}$ for fixed $\rho$

$$
\left(e^{-\tau}-\sin \rho \frac{1+r^{2}}{1-r^{2}}\right)^{2}+\cos ^{2} \rho\left(\frac{1+r^{2}}{1-r^{2}}\right)^{2}-\frac{4 r^{2}}{\left(1-r^{2}\right)^{2}}=0
$$

Solving equation above we obtain

$$
e^{-\tau}=\sin \rho \frac{1+r^{2}}{1-r^{2}} \pm \sqrt{\frac{4 r^{2}}{\left(1-r^{2}\right)^{2}}-\cos ^{2} \rho\left(\frac{1+r^{2}}{1-r^{2}}\right)^{2}}
$$

with solutions existing for $\rho$ such that

$$
|\cos \rho| \leq \frac{2 r}{1-r^{2}} \frac{1-r^{2}}{1+r^{2}}=\frac{2 r}{1+r^{2}} .
$$

The maximal value for $e^{-\tau}$ is achieved at $\rho=\frac{\pi}{2}$ and it is

$$
e^{-\tau}=\frac{1+r^{2}}{1-r^{2}}+\frac{2 r}{1-r^{2}}=\frac{1+r^{2}+2 r}{1-r^{2}}=\frac{(1+r)^{2}}{1-r^{2}}=\frac{1+r}{1-r} .
$$

Therefore the maximal value for $|s|$ is achieved at $\rho=\frac{\pi}{2}$, is equal to $|s|=\log \left(\frac{1+r}{1-r}\right)$, and for $r=1-\delta$ we have the maximal value

$$
\max |s|=\log \left(\frac{1+r}{1-r}\right)=-\log \delta+\log (2-\delta) .
$$


Since function $D_{N}(s)$ has no zeros in $\left\{s: \sigma_{1}<\operatorname{Re} s<\sigma_{2}\right\}$ we can consider analytic function $\log \left(D_{N}(s)\right)$ in this strip, and using estimates (35) and (31), and equality (42), we obtain the following estimate for $z=(1-\delta) e^{i \theta}$

$$
\log \left|D_{N}\left(\Psi^{-1}(z)\right)\right| \leq \frac{\left\|N_{\Psi^{-1}(z)}\right\|^{2}}{2} \leq C\left|\Psi^{-1}(z)\right|^{4} \leq C|\log \delta|^{4} .
$$

Using then the Borel-Caratheodory inequality ([Ti1], [Boa]) on disks with radii

$$
1-2 \delta=r<R=1-\delta,
$$

we obtain

$$
\begin{gathered}
\left|\log \left(D_{N}\left(\Psi^{-1}(z)\right)\right)\right|_{\{|z|=1-2 \delta\}} \\
\leq \frac{2-4 \delta}{\delta} \max _{|z|=R} \operatorname{Re}\left\{\log \left(D_{N}\left(\Psi^{-1}(z)\right)\right)\right\}+\frac{1-\delta+1-2 \delta}{\delta}\left|\log \left(D_{N}\left(\Psi^{-1}(0)\right)\right)\right| \\
<\frac{C}{\delta} \log ^{4} \delta
\end{gathered}
$$

or

$$
-\frac{C}{\delta} \log ^{4} \delta<\left.\operatorname{Re}\left\{\log \left(D_{N}\left(\Psi^{-1}(z)\right)\right)\right\}\right|_{\{|z|=1-2 \delta\}}<\frac{C}{\delta} \log ^{4} \delta
$$

From the last estimate we obtain an estimate for the function $\left|1 / D_{N}\left(\Psi^{-1}(z)\right)\right|$ in the disk $\mathbb{D}(1-2 \delta)$ :

$$
\left.\left|1 / D_{N}\left(\Psi^{-1}(z)\right)\right|\right|_{\{|z| \leq 1-2 \delta\}}<C \exp \left\{\frac{|\log \delta|^{4}}{\delta}\right\}
$$

for arbitrary $\epsilon>0$.

For a fixed $\rho \in(0, \pi)$ and arbitrary $\tau$ we have that $\rho+i \tau \in \Psi^{-1}(\mathbb{D}(r))$ with $r=1-2 \delta$ if

$$
\begin{gathered}
e^{|\tau|} \leq \sin \rho \cdot \frac{1+r^{2}}{1-r^{2}}+\sqrt{\frac{4 r^{2}}{\left(1-r^{2}\right)^{2}}-\cos ^{2} \rho \cdot\left(\frac{1+r^{2}}{1-r^{2}}\right)^{2}} \\
=\sin \rho \cdot \frac{2-4 \delta+4 \delta^{2}}{2 \delta(2-2 \delta)}+\frac{\sqrt{4(1-2 \delta)^{2}-\cos ^{2} \rho \cdot\left(2-4 \delta+4 \delta^{2}\right)^{2}}}{2 \delta(2-2 \delta)},
\end{gathered}
$$

and therefore for any interval $\left[\gamma^{\prime}, \pi-\gamma^{\prime}\right]$ there exist constants $C_{1}, C_{2}$ such that conditions

$$
\rho \in\left[\gamma^{\prime}, \pi-\gamma^{\prime}\right], \quad \frac{C_{1}}{\delta}<e^{|\tau|}<\frac{C_{2}}{\delta}
$$

imply that $\rho+i \tau \in \Psi^{-1}(\mathbb{D}(1-2 \delta))$.

Using then estimate (43) we obtain for $s$ with $\operatorname{Re} s \in\left[\sigma_{1}+\frac{\gamma^{\prime}\left(\sigma_{2}-\sigma_{1}\right)}{\pi}, \sigma_{2}-\frac{\left(\pi-\gamma^{\prime}\right)\left(\sigma_{2}-\sigma_{1}\right)}{\pi}\right]$ estimate

$$
\left|1 / D_{N}(s)\right|<\exp \left\{C e^{|s|} \cdot(1+|s|)^{4}\right\}
$$

for arbitrary $\epsilon>0$, which leads to estimate (41).

Combining now estimate (40) for $\left\|D_{N}\left(\begin{array}{l}x \\ y\end{array} \mid s\right)\right\|$ with estimate (41) we obtain estimate (39). 


\section{Proof of Theorem 1.}

According to Lemmas 2.1 and 2.2 any function defined by formula (5) satisfies equation (1). Also, as we pointed out earlier, this function will automatically satisfy boundary condition (3). Therefore, to prove Theorem 1 we have to solve integral equation (18) for the function $p(\xi, s)$, so that the boundary condition (2) will be also satisfied. As in Proposition 5.3 in order to solve equation (18) we first solve equation (29)

$$
\mathcal{G}_{s}\left[r_{s}\right]=\mathcal{R}_{s} \circ \mathcal{T}^{-1}\left[r_{s}\right]=-\frac{i U}{2\left(1-M^{2}\right)^{3 / 2}} \widehat{w}(x, s) e^{-s c x}
$$

and then define

$$
p(\xi, s)=\mathcal{T}^{-1}\left[r_{s}\right] .
$$

Using Proposition 6.1 we obtain that the solution of (29) is defined by the formula

$$
r_{s}=\left(\mathcal{I}-\mathcal{H}_{s}\right)\left[-\frac{i U}{2\left(1-M^{2}\right)^{3 / 2}} \widehat{w}(x, s) e^{-s c x}\right],
$$

where $\mathcal{H}_{s}$ is an integral operator with kernel

$$
H_{s}(x, y)=\left[D_{N_{s}}\right]^{-1} \cdot D_{N}\left(\begin{array}{c|c}
x & s \\
y & s
\end{array}\right)
$$

admitting estimate (39). Combining estimate (39) with estimate (4) we obtain estimate

$$
\|r(\cdot, \sigma+i \nu)\|_{L^{2}[-1,1]}<C
$$

with constant $C$ independent of $s$. Then from Proposition 5.1 we obtain estimate (6) for $p(\xi, s)=\mathcal{T}^{-1}\left[r_{s}\right]$.

\section{REFERENCES}

[A] N.I. Akhiezer, On some inversion formulas of singular integrals, Izv. Akad. Nauk SSSR, v.9 (1945), 275-290.

[AG] N.I. Akhiezer, I.M. Glazman, Theory of linear operators in Hilbert space, v.I, Dover, New York, 1993.

[Ba1] A.V. Balakrishnan, Semigroup theory in aeroelasticity, Progress in Nonlinear Differential Equations and Their Applications, v.42, 15-24, Birkhäuser Verlag, 2000.

[Ba2] A.V. Balakrishnan, Possio integral equation of aeroelasticity theory, Journal of Aerospace Engineering, 16:4 (2003), 139-154.

[BAH] R.L. Bisplinghoff, H. Ashley, R.L. Halfman, Aeroelasticity, Dover, New York, 1996.

[Boa] R. Boas, Entire functions, Academic Press, New York, 1954.

[Boc] S. Bochner, Lectures on Fourier integrals, Princeton University Press, Princeton, NJ, 1959.

[C] T. Carleman, Zur Theorie der linearen Integralgleichungen, Math. Zeitschrift, v. 9, 196-217, 1921.

[EMOT] A. Erdélyi, W. Magnus, F. Oberhettinger, F.G. Tricomi, v.I Tables of integral transforms, v.II Higher transcendental functions, CalTech Bateman Manuscript Project, McGraw-Hill, 1954.

[GR] I.S. Gradshteyn, I.M. Ryzhik, Table of integrals, series, and products, Academic Press, 1994.

[L] P.D. Lax, Functional analysis, John Wiley \& Sons, 2002.

[LL] L.D. Landau, E.M. Lifshitz, Fluid Mechanics, Pergamon Press, 1959.

[M] S.G. Mikhlin, Integral equations, Pergamon Press, 1957.

[P] P.L. Polyakov, On a boundary value problem in subsonic aeroelasticity and the cofinite Hilbert transform, Preprint, 2004, http://www.arxiv.org/math.AP/0406191.

[RS] M. Reed, B. Simon, Functional Analysis, Academic Press, 1980.

[Se] L.I. Sedov, Two-dimensional problems in hydrodynamics and aerodynamics, Interscience Publishers, 1965, (Russian edition 1950).

[So] H. Söhngen, Die Lösungen der Integralglechung und deren Anwendung in der Tragflügeltheorie, Math. Zeitschrift, v. 45, 245-264, 1939.

[St] E. Stein, Singular integrals and differentiability properties of functions, Princeton University Press, Princeton, NJ, 1970.

[Ti1] E.C. Titchmarsh, The theory of functions, Oxford University Press, 1939.

[Ti2] E.C. Titchmarsh, Introduction to the theory of Fourier integrals, Chelsea, New York, 1986.

[Tr] F.G. Tricomi, Integral equations, Intersciense Publishers, New York, 1957.

Department of Mathematics, University of Wyoming, Laramie, WY 82071, USA

E-mail address: polyakov@uwyo.edu 